\title{
Palivizumab for prophylaxis of respiratory syncytial virus infections in preterm neonates
}

\author{
Prophylaxe von Infektionen durch das respiratorische Synzytialvirus bei \\ Frühgeborenen
}

\author{
Korrespondenzadresse \\ Ludwig Gortner \\ Klinik für Kinder- und Jugendheilkunde \\ Medizinische Universität Wien \\ Währinger Gürtel 18-20 \\ 1090 WIEN \\ Österreich \\ ludwig.gortner@meduniwien.ac.at
}

\author{
Bibliografie \\ DOI http://dx.doi.org/10.1055/s-0043-116334 \\ Klin Padiatr 2017; 229: 259-260 \\ (c) Georg Thieme Verlag KG Stuttgart · New York \\ ISSN 0300-8630
}

Recent data indicate that the number of children cared for respiratory syncytial virus (RSV) infections in hospitals is in the range of 50,000 to 100,000 per year in the United States [3]. Major risk factors include apart from prematurity specifically below 29 weeks, congenital heart defects, Down syndrome and further congenital anomalies. The American Academy of Pediatrics recommended in 2014 a restriction of Palivizumab prophylaxis to preterm infants of $<29$ weeks of gestation and those with a high-risk course postnatally and higher gestational ages apart from specific indications resulting from congenital anomalies including congenital heart defects [1].

In the current issue of the Klinische Pädiatrie, a group from Bosnia-Herzegovina published data on the effects of prophylactic immunization with Palivizumab on the occurrence of RSV infection in preterm neonates and other high-risk children [6].

Main data of the present study indicate that out of 101 children about $49 \%$ were preterm neonates, $40 \%$ had a congenital heart defect and $11 \%$ exhibit individual risk factors. The authors showed clearly that out of 101 children, who were treated prophylactically with Paliviziumab, none was hospitalized due to RSV infection. Despite the small number of the enrolled infants and older children, the data indicate that also under conditions of a reduced economical state of the National Health Service, the prophylaxis is working adequately.

However, there still remain some questions open with respect to efficacy of Palivizumab.

1. The number of preterm neonates being enrolled in the trial was relatively low, thus, giving only a restricted measurement of effectiveness of Palivizumab.

2. The data have to be interpreted with caution, since the effects of Palivizumab have been restricted to somewhat less than $50 \%$ of preterm neonates, the further treated children of the group are either affected by congenital heart disease or by other disorders, e. g. neurologic problems, cardiopulmonary anomalies among others.
In a recently published multicenter analysis enrolling pediatric hospitals from the Northern as well as from the Southern hemisphere (United States, Canada, Italy, The Netherlands and Australia), infants were enrolled, if they were born prematurely ( $<36$ weeks of gestation) and were younger than 24 months. In the second year of life, only infants with the diagnosis of bronchopulmonary dysplasia (BPD) or related pulmonary disorders were enrolled [3]. Out of more than 1,000 preterm neonates enrolled in this study, about 900 were able to be identified as the group with adequate data available. Main results of this study include that Palivizumab was used in about 430 infants, whereas it was not given in 415 neonates serving as controls. RSV was identified in $47 \%$ on occasion of either outpatient or hospitalized care in enrolled children with respiratory symptoms. RSV was identified in a total of 403 neonates, the effect of Palivizumab for intensive care admission was a risk reduction $58 \%(\mathrm{Cl} 43-69 \%$ on the $95 \%$ level) for neonates of 24 to 35 weeks of gestation without chronic lung disease (BPD) and other children enrolled in the trial after adjustment for common risk factors; for children having a gestational age of 28 to 35 weeks of gestation without BPD it was $74 \%$ (Cl 56-85\% on the $95 \%$ level). However, in this trial, no data on the severity of respiratory symptoms of hospitalized children as well as on mortality were published [3].

The authors discuss a high-degree efficacy rate of Palivizumab also in children of $>29$ weeks of gestation and thus propose in order to redefine the indications for Palivizumab in the prophylaxis of less immature premature infants related to the indications proposed by the American Academy of Pediatrics. Recent data thus confirm older study results and indicate that the outcome of RSV infections is improved by RSV prophylaxis using Palivizumab in neonates $>29$ weeks of gestation, a clear-cut reduction in mortality has not been proven until now [1,2].

In the context of data published from a German group [8] where the authors also could show that there is a reduction of RSV related hospitalization after Palivizumab among preterm neonates of a birthweight below $1,500 \mathrm{~g}$, however, also without changing of mortality. These data further add some weight with respect to preven- 
tion of RSV with respect to the number of hospitalized preterm neonates, however, also without affecting mortality.

Interestingly, in these data, the hospitalization rate of infants receiving the immunoprophylaxis according to the national guidelines already during the primary hospitalization period - without Palivizumab - also showed a reduction in the number of preterm neonates, who needed hospitalization from RSV infections. At least with respect to the data following Palivizumab, however, there is a constant effect in older infants than 29 weeks of gestation with respect to prevention of hospitalization. This also includes children, who need intensive care due to RSV induced obstructive lung disease.

What do these data mean for further procedure on RSV prophylaxis?

In the eyes of the author of this editorial, the data for less immature preterm neonates with a gestational age of more than 29 weeks gestational age, should further be collected and put together in a metaanalysis on the effects of Palivizumab on RSV infections, which need to be treated under hospitalized conditions. Additionally, long-term outcome variables up to school age should further be carried out in order to address the question, if the partial avoidance of RSV infections during the first two years of life translates into an improved lung function during this period compared to those children without RSV prophylaxis.

Further questions remain with respect to other indications than prematurity associated risk factors for RSV infections. These include neonates, who were treated for congenital heart disease during the first months of life where the efficacy is adequate for giving Palivizumab in severe cases of congenital heart disease.

Further indications include children with Down syndrome, a group, which has been focused mainly during the last five years. A recent metaanalysis indicates that in infants with Down syndrome, irrespectively of prematurity and congenital heart disease, there is an effect in reducing hospitalizations due to RSV [7].

Apart from the above-mentioned risk cohorts, infants and older children with cystic fibrosis have been found to be significantly threatened for severe RSV infections. Thus, studies have been performed mainly during the last about 10 years with administration of Palivizumab during the first year of life. However, no data on clearcut effects of the prophylaxis in children with cystic fibrosis have been established. Thus, the analysis of the Cochrane group resumes for children with cystic fibrosis that there is no clear-cut indication for the prophylaxis with Palivizumab as only one controlled clinical trial was included into the analysis due to methodological problems of other trials, which had been published before [5].

The study enrolled in the Cochrane trial is from Northern Ireland and showed that the earlier time period of the first isolate of pseudomonas aeroguinosa was seen in children, who were submitted to a Palivizumab prophylaxis [5]. However, no data on the further course are given, so, this finding has to be regarded with some caution.

With respect to preterm neonates, who are the focus of this editorial, prophylaxis with Palivizumab should be one point in the follow-up care of these infants. Clear-cut data from prospective trials for modifying these recommendations given by the American Academy of Pediatrics do not appear to have been published at the moment. There is some degree of discussion, as to further concepts in post-hospitalization period of very preterm infants should be introduced [4].

Apart from these reflections, prophylaxis of viral infections overall during the first two years in very preterm neonates seem to be mandatory. Thus, RSV prophylaxis only represents one point for extremely and very immature preterm neonates, who are cared for at home after the discharge from hospital.

\section{Literatur}

[1] Updated guidance for palivizumab prophylaxis among infants and young children at increased risk of hospitalization for respiratory syncytial virus infection. Pediatrics 2014; 134: e620-e638

[2] Alan S, Erdeve O, Cakir U et al. Outcome of the Respiratory Syncytial Virus related acute lower respiratory tract infection among hospitalized newborns: a prospective multicenter study. The journal of maternal-fetal \& neonatal medicine: the official journal of the European Association of Perinatal Medicine, the Federation of Asia and Oceania Perinatal Societies, the International Society of Perinatal Obstet 2016; 29: 2186-2193

[3] Anderson E], Carosone-Link P, Yogev R et al. Effectiveness of Palivizumab in High-risk Infants and Children: A Propensity Score Weighted Regression Analysis. The Pediatric infectious disease journal 2017; 36: 699-704

[4] Forster KM, Immler S, Ensslen M et al. Indications and concept of follow-up care of home-monitoring for premature and risk infants. Klin Padiatr 2015; 227: 72-79

[5] Groves HE, Jenkins L, Macfarlane M et al. Efficacy and long-term outcomes of palivizumab prophylaxis to prevent respiratory syncytial virus infection in infants with cystic fibrosis in Northern Ireland. Pediatr Pulmonol 2016; 51: 379-385

[6] Jerkovic Raguz M, Grgic I, Brzica J. Palivizumab: The effects of prophylactic immunization on the occurrence of infections caused by the Respiratory Syncytial Virus. Klin Padiatr 2017; 229:

[7] Manzoni P, Figueras-Aloy J, Simoes EAF et al. Defining the Incidence and Associated Morbidity and Mortality of Severe Respiratory Syncytial Virus Infection Among Children with Chronic Diseases. Infect Dis Ther 2017, doi:10.1007/s40121-017-0160-3

[8] Stichtenoth G, Hartel C, Spiegler J et al. Increased risk for bronchitis after discharge in non-vaccinated very low birth weight infants. Klin Padiatr 2015; 227: 80-83 